\title{
Myroides spp. Causing Urinary Tract Infection in an Immunocompromised Patient: A Case Report
}

\author{
Nivedita Thass", Manoj Kumar and Ravinder Kaur
}

Department of Microbiology, Lady Hardinge Medical College, New Delhi-110001, India

*Corresponding author

\section{A B S T R A C T}

\section{Keywords \\ Myroides, Urinary tract infection, \\ Immunocompromised, \\ Multidrug resistant \\ Article Info \\ Accepted: \\ 10 March 2018 \\ Available Online: \\ 10 April 2018}

Myroides spp. have historically been rare opportunistic pathogens although present widely in nature. Their notorious resistance to most classes of antibacterials and invasive potential leading to serious infections among the immunocompromised population calls for appropriate testing and management of the infections caused by Myroides spp.

\section{Case Study}

A 12 year old girl presented to the paediatric OPD with complains of generalised swelling and decreased urine output for the past ten days. The swelling started from the face followed by the abdomen and limbs. The patient also had cough, difficulty in breathing and episodes of vomiting for the past 2 days. The vomiting was non-bilious and nonprojectile. There was no history of fever, pain abdomen, loose stools, burning micturition or haematuria. There was no history of headache, or any evidence of focal neurological defect. On examination her blood pressure was found to be raised. Her cardiovascular and motor system functions were found to be within normal limits. On per abdomen examination, abdomen was found to be soft and distended, no organomegaly found and bowel sounds were normal. Her haemogram was within normal limits, ANA was negative. Chest Xray showed signs of pleural effusion and pleural fluid was negative for $\mathrm{TB}$ by Genexpert. Her C3 and C4 complement levels were within normal limits. On urine analysis showed protein to creatinine ratio of 2.57 . Urine dipstick showed- albumin (3+) and sugar (nil). Serum protein was 3.5-4 g/dl (normal: 6-8.3 g/dl) and serum albumin 1.3$1.5 \mathrm{~g} / \mathrm{dl}(3.5-5 \mathrm{~g} / \mathrm{dl})$. Her total blood cholesterol was $912 \mathrm{mg} / \mathrm{dl}$ (normal <200 $\mathrm{mg} / \mathrm{dl}$ ). The patient had hypoalbuminemia, hyper albuminuria, hyperlipidemia and generalised edema, thus a presumptive diagnosis of nephrotic syndrome was made. 
She was started on tab. Furosemide, tab. Wysolone, tab. Lasix and inj. Ceftriaxone. On day 6 of her admission, she received albumin infusion. The patient was kept on a high protein diet. The patient was stable and responding to the treatment. However on day 22 of her admission, the patient had a seizure and was shifted to the ICU. The patient was started on tab. Phenytoin. The patient continued to have decreased urine output and she started to develop edema again. Over the next 24 hours patient also developed fever. Urine routine and microscopy was done. It again showed hyper albuminuria, also this time patient had pyuria (5-10 pus cells/hpf) and microscopy revealed presence of bacilli. Urine sample was sent for culture.

Culture revealed non-lactose fermenting, smooth, non-mucoid colonies measuring 2-3 $\mathrm{mm}$ on CLED agar and blood agar incubated under aerobic conditions showed round, convex, smooth, yellowish colonies measuring 1-2 mm. Gram stain showed gram negative bacilli. The organism was non-motile, indole negative, oxidase and catalase positive. The organism was identified as Myroides spp. By VITEK 2 system with an identification score of $96 \%$. Unfortunately species level identification could not be done.

The antimicrobial testing was done using Muller Hinton agar by Kirby-Bauer disc diffusion method using CLSI guidelines 2016. Zone diameters for Pseudomonas were used as no standards are available for Myroides spp. The isolate was found to be resistant to all antimicrobials (Table 1). The MIC was obtained by VITEK 2 system and the organism was resistant to all antibiotics except Minocycline and intermediate susceptibility was seen for Tigecycline (Table 2).

The patient was started on inj. minocycline. On day 3 of starting minocycline her urine output started increasing. Further management of nephrotic syndrome continued with steroids and high protein diet.

\section{Results and Discussion}

Members of the genus Myroides were first isolated by Stutzer in 1923 and classified them as Bacterium faecale aromaticum. Later, in 1929 Stutzer and Kwaschnina, renamed it as Flavobacterium odoratus (Elantamilan et al., 2015). In 1996on the basis of extensive polyphasic taxonomic analysis it was determined that the organism formerly classified as Flavobacterium odoratum consisted of a heterogeneous group comprising of Myroides odoratus and Myroides odoratimimus (Vancanneyt et al., 1996). Genus Myroides are not part of normal human microflora, rather they are encountered in the wet environments, sea water, soil and sewage treatment plants (Manish Rajan et al., 2017). Over the last few years this organism has been reported to be associated with various life threatening infections including recurrent cellulitis, post-operative wound infections and severe septicaemia (Elantamilan et al., 2015). They are mostly low grade opportunistic pathogens, infecting immunocompromised hosts such as those suffering from liver cirrhosis, end stage renal disease and chronic obstructive pulmonary disease on long term corticosteroid therapy (Beharrysingh, 2017). A case of Myroides odoratimimus bloodstream infection in a patient with chronic diabetic foot ulcer has been reported (Endocott Yazdani et al., 2015). Another report documented a case of soft tissue infection, septic shock and pneumonia due to $M$. odoratimimus in an immunocompetent male (Benedetti et al., 2011). Few cases of central venous catheter blood stream infections, soft tissue infections, endocarditis and ventriculitis have also been reported due to Myroides spp. (Ferrer et al., 1995; Mac Farlane et al., 1985; Motwai et al., 2004). 
Table.1 Kirby Bauer disc diffusion method on Muller Hinton agar showing inhibition zone size for antibacterials

\begin{tabular}{|l|l|}
\hline Antimicrobial agent & Zone of inhibition (mm) \\
\hline Piperacillin- tazobactam & 12 \\
\hline Norfloxacin & No zone \\
\hline Amikacin & No zone \\
\hline Nitrofurantoin & No zone \\
\hline Trimethoprim-sulphamethoxazole & No zone \\
\hline Colistin & No zone \\
\hline Ertapenem & No zone \\
\hline
\end{tabular}

Table.2 MICs of antimicrobial agents obtained using the VITEK 2 system

\begin{tabular}{|l|l}
\hline Antimicrobial agent & MIC $(\mu \mathrm{l} / \mathrm{ml})$ \\
\hline Piperacillin tazobactam & $>=128$ \\
\hline Ticarcillin/clavulanic acid & $>=128$ \\
\hline Ceftaridime & $>=64$ \\
\hline Cefoperazone/ sulbactam & $>=64$ \\
\hline Cefepime & $>=64$ \\
\hline Aztreonam & $>=64$ \\
\hline Imipenem & $>=16$ \\
\hline Meropenem & $>=16$ \\
\hline Amikacin & $>=64$ \\
\hline Gentamicin & $>=16$ \\
\hline Ciprofloxacin & $>=4$ \\
\hline Levofloxacin & $>=8$ \\
\hline Minocycline & $<=1$ \\
\hline Tigecycline & 4 \\
\hline Colistin & $>=16$ \\
\hline Trimethoprim-sulphamethoxazole & $<=320$ \\
\hline
\end{tabular}

Two studies reported nosocomial outbreaks of urinary tract infections in the setting of urinary stones or cancer (Ktari et al., 2012; Yagci et al., 2000). In nosocomial infections the source is mostly unknown, however water has been suspected to carry this organism (Hugo et al., 2006). Animal bites have also been implicated as one of the sources of infections (Maraki et al., 2012). Very few cases of infections due to Myroides spp. has been published in India till date. A case of septicaemia from Shillong (Elantamilan et al.,
2015), case of urosepsis from Pune(Manish Ranjan et al., 2017) and a case of pericardial effusion from Uttarakhand (Prateek et al., 2015) due to Myroides spp. have been reported in the past. Here we report a case of urinary tract infection due to Myroides spp. in a child suffering from nephrotic syndrome. Immunocompromised status of the patient due to prolonged hospitalisation and treatment with steroids could have been the reasons why the patient got infected with this rare pathogen. 
Treatment of Myroides spp. infections is often difficult as they have been found to harbour a KPC-2 carbapenemase, which can hydrolyse expanded-spectrum cephalosporins and carbapenems (Kuai et al., 2011). The production of chromosome encoded metallob-lactamases has also been documented in both $M$. odoratus (TUS-1) and $M$. odoratimimus (MUS-1) (Mammeri et al., 2002). Majority of the strains are resistant to beta-lactams, including aztreonam and carpenems, they exhibit variable susceptibility to aminoglycosides, quinolones and trimethoprim-sulphamethoxazole. Our patient showed susceptibility for Minocycline and intermediate susceptibility for Tigecycline. Patient responded well to minocycline. However, she remained hospitalised for further management of Nephrotic syndrome.

Empirical therapy is usually ineffective due to the multidrug resistance found in Myroides spp. Physicians thus need to carefully consider this organism as a pathogen among the immunocompromised patients. This can lead to timely intervention and prompt switching to definitive treatment of infections caused by Myroides spp.

\section{References}

Beharrysingh, R. (2017). Myroides bacteremia: A case report and concise review. IDCases 8: 34-36.

Benedetti, P., Rassu, M., Pavan, G., Sefton, A. \& Pellizzer, G. (2011). Septic shock, pneumonia, and soft tissue infection due to Myroides odoratimimus: report of a case and review of Myroides infections. Infection 39, 161-165.

CLSI. (2016). M100S, Performance Standards for Antimicrobial Susceptibility Testing. 26th edition, Clinical and Laboratory Standards Institute, USA: supplement M100S.
Deepa, R., Venkatesh, K. G., Parveen, J. D., Banu, S. T. and Jayalakshmi, G. (2014). Myroidesodoratus and Chryseobacterium indologenes: two rare isolates in the immunocompromised. Indian $\mathrm{J}$ Med Microbiol 32, 327-330.

Elantamilan, D., B. Choudhury, A. B. Khyriem, V. W. Lyngdoh and J. Rajbongshi (2015). Septicaemia caused by Myroides spp.: a case report. JMM Case Reports 2(5).

Endicott-Yazdani T.R., Dhiman N., Benavides R., Spak C.W. Myroides odoratimimus bacteremia in a diabetic patient. Proc (Bayl Univ Med Cent) 2015; 28(3):342-343

Ferrer C, Jakob E, Pastorino G, Juncos LI. Right-sided bacterial endocarditis due to Flavobacteriun odoratum in a patient on chronic haemodialysis. Am J Nephrol. 1995; 15:82-84.

Hugo, C. J., Bruun, B. \& Jooste, P. J. (2006). The genera Empedobacter and Myroides. In The Prokaryotes, pp. 532538. Edited by M. Dworkin, S. Fallow, E. Rosenberg, K.-H. Schleifer \& E. Stackebrandt. New York: Springer.

Ktari, S., Mnif, B., Koubaa, M., Mahjoubi, F., Ben Jemaa, M., Mhiri, M. N. \& Hammami, A. (2012). Nosocomial outbreak of Myroides odoratimimus urinary tract infection in a Tunisian hospital. J Hosp Infect 80, 77-81

Kuai, S., Huang, L., Pei, H., Chen, Y. \& Liu, J. (2011). Imipenem resistance due to class A carbapenemase KPC-2 in a Flavobacterium odoratum isolate. J Med Microbiol 60, 1408-1409.

MacFarlane DE, Baum-Thureen P, Crandon I. Flavobacteriun odoratum ventriculitis treated with intraventricular cefotaxime. J Infect. 1985; 11:233-38.

Mammeri, H., Bellais, S. and Nordmann, P. (2002). Chromosome encoded blactamases TUS-1 and MUS-1 from 
Myroides odoratus and Myroides odoratimimus (formerly Flavobacterium odoratum), new members of the lineage of molecular subclass B1 metalloenzymes. Antimicrob Agents Chemother 46, 3561-3567

Manish Ranjan, Santosh Karade, Praveen Rahi, S.P. Singh and Sourav Sen. 2017. Urosepsis due to Multi Drug Resistant Myroides odoratimimus: A Case Report. Int.J.Curr.Microbiol.App.Sci. 6(8): 1930-1935.

Maraki, S., Sarchianaki, E. and Barbagadakis, S. (2012). Myroides odoratimimus soft tissue infection in an immunocompetent child following a pig bite: case report and literature review. Braz J Infect Dis 16, 390-392.

Motwai B, Krezolek D, Symeonides S, Khayr W. Myroides odoratum cellulitis and bacteraemia: a case report. Infect Dis Clin Pract. 2004; 12:343-44.

Prateek S., Gupta P., Mittal G., Singh A.K. Fatal case of pericardial effusion due to
Myroides odoratus: a rare case report. J Clin Diagn Res. 2015; 9(11):DD01-2.

$\mathrm{Su}$, Mei-Yu et al., Urinary tract infection caused by Myroides species: A case report Journal of Microbiology, Immunology and Infection, Volume 48, Issue 2, S133.

Vancanneyt M, Segers P, Torck U, Hoste B, Bernardet J-F, Vandamme $\mathrm{P}$, et al., Reclassification of Flavobacterium odoratum (Stutzer 1929) strains to a new genus, Myroides, as Myroides odoratus comb. Nov. and Myroides odoratimimus sp. nov. Int $\mathbf{J}$ Syst Bacteriol. 1996; 46:926-32.

Yag ci, A., Cerikc,iog lu, N., Kaufmann, M. E., Malnick, H., So“ yletir, G., Babacan, F. and Pitt, T. L. (2000). Molecular typing of Myroides odoratimimus (Flavobacterium odoratum) urinary tract infections in a Turkish hospital. Eur J Clin Microbiol Infect Dis 19, 731-732.

\section{How to cite this article:}

Nivedita Thass, Manoj Kumar and Ravinder Kaur. 2018. Myroides spp. Causing Urinary Tract Infection in an Immunocompromised Patient: A Case Report. Int.J.Curr.Microbiol.App.Sci. 7(04): 997-1001. doi: https://doi.org/10.20546/ijcmas.2018.704.108 